Espacio, Tiempo y Forma, Serie Il, Historia Antigua, t. 9, 1996, págs. 289-319

\title{
La religión practicada por los militares del Ejército Romano de Hispania durante el Alto Imperio Romano (ss. I-III)
}

\author{
Luis Sagredo San Eustaquio* y Agustín Jiménez de Furundarena
}

\section{INTRODUCCIÓN}

El estudio de la religión practicada en las unidades del ejército romano ha sido siempre un tema que ha apasionado a los investigadores del mundo romano, disponiendo de una serie de obras generales referidas a este apartado de la vida militar durante el Imperio ${ }^{1 .}$

Sin embargo, la religión practicada por el ejército romano acuartelado en Hispania durante el Alto Imperio (siglos I-III) no ha sido, hasta el presente, analizada de una forma monográfica ${ }^{2}$.

La base de trabajo para poder realizar esta investigación se encuentra en los testimonios que sobre el mundo religioso proporciona la epigrafía romana de la Península, ya que son las inscripciones la fuente principal, casi única, para estudiar la religión de los militares que sirvieron en Hispania. A ellas se añaden algunos testimonios de tipo arqueológico, procedentes de las escasas e incompletas excavaciones arqueológicas de los campamentos donde estuvieron asentadas las unidades con guarnición en la Península Ibérica, fundamentalmente Petavonium (Rosinos de Vidriales, Zamora) y Legio VII Gemina (León).

\footnotetext{
* Universidad de Valladolid.

1 A. Von Damazewski.: Die Fahnen im Römische Heere. Viena 1885. Ídem. "Die Religion des Römischen Heeres». Westdeutsche Zeitechrift für Geschichte un Kust, Trier, 1895, pp. 1-128 (= "Aflfsätze zur Römischen Heeresgeschichte» Darmstadt, 1972, pp. 81-209). G.R. WATSON: The Roman Imperial Soldier. Londres, 1969, pp. 127-133. H. ANKERSDORFER: Studien zur Religion des Römisches Heeres von Augustas bis Diokletian. Diis. Constanza 1973. E. BIRLEY. "The Religion of the Roman Army: 1885-1977". ANRW, II, 16.2, 1978, pp. 1506-1541. J. HELGELAND. «Roman Army Religion». ANRW, II, 16.2, 1978, pp. 1470-1505. Y. LE BOHEC: L'Armée Romaine. París 1989, pp. 252-267.

2 Sólo P. LE Roux: L'Armée Romaine des Provinces lbériques d'Auguste à l'Invasion de 406. París 1982, dedica unas breves páginas a estudiar sumariamente la religión del ejército romano de Hispania.
} 
En el Imperio Romano, la inmensa mayoría de la población practicaba uno $u$ otro tipo de religión, ya que el mundo romano tenía completamente sacralizados todos y cada uno de los aspectos de la vida. La Religión en el mundo de la milicia, al igual que en el mundo civil, trataba de proveer a los creyentes de un marco de referencia en el que estuvieran inscritos todos los aspectos de su vida social y personal ${ }^{3}$.

El Imperio, hasta el triunfo definitivo del cristianismo con Teodosio a finales del siglo IV, nunca tuvo como tal una religión "oficial», aunque las formas de culto relacionadas con la religión cívica tradicional de Roma, heredadas de la Monarquía y la República, y el culto imperial, se extendieron por todos los rincones de su territorio y debieron ser respetadas de forma universal, pero los habitantes del Imperio no tenían obligación de creer en ellas.

El ejército, en este sentido, era una excepción, ya que en él esta religión cívica y el culto imperial eran una verdadera religión oficial, en la que los militares debían creer obligatoriamente. Ello no impedía que los soldados tuvieran otras particulares creencias, pero dentro de los campamentos la única religión admitida y pública era la que se consideraba oficial.

Esta imposición religiosa estaba relacionada con la disciplina y con la creación en cada unidad y en el ejército en general de un sprit de corps, que se apoyaba sobre una unidad de acción y mando procedentes del entrenamiento y la jerarquía, y que se veian reforzadas con una unidad, una uniformidad de formas de vida, hasta tal punto que los campamentos eran auténticas islas de romanidad en medio de un mar de costumbres no romanas, a lo que se sumaba la imposición del latín como única lengua militar ${ }^{4}$. Para completar estas dos realidades, consciente o inconscientemente, se intentó también una unidad de creencias públicas que fuera común a todo el ejército y que coayudaran a forjar ese sprit de corps ${ }^{5}$.

De esta manera, al iguai que la disciplina, la lengua, y las formas de vida romanas, la religión oficial del ejército intentaba que los soldados tuvieran un marco de referencia claro que les enseñara cual era su papel dentro de su mundo, que muy pocos dudaban que estuviera regido por los dioses ${ }^{6}$.

\footnotetext{
J. HeLGeLAND: «Roman Army Religion...», pp. 1.471-1.472.

G.R. WATSON: The Roman Imperial Soldier..., pp. 38 y 41.

J. HELGELAND: «Roman Army Religion...», p. 1.473.

J. Helgeland: «Roman Army Religion...», p. 1473.
} 
La misión última de la religión era, pues, asegurar a los soldados que su profesión era coherente con el destino del Imperio y de los dioses, y que, en última instancia, nunca iban a ser abandonados ni sacrificados inútilmente ni por sus camaradas, ni por sus jefes, ni por el emperador, ni por los dioses de Roma ?

Así, como demuestra el Feriale Duranum ${ }^{8}$, los actos del culto oficial del ejército romano formaban parte de la vida cotidiana de los soldados con el mismo rango que la obediencia, la disciplina, el entrenamiento o el entretenimiento. En realidad, esto significaba que toda la vida militar se encontraba impregnada de un halo religioso y era en sí misma parte de un sistema religioso.

Esta inmersión religiosa comenzaba desde el mismo instante en que el recluta entraba en el campamento, ya que lo primero que debían hacer, y que haría todos los días durante su permanencia en filas, era pronunciar la formula del sacramentum o juramento de lealtad al emperador y a los dioses del Imperio ${ }^{9}$. Su nombre ya es altamente significativo - sacramentum, de sacratio, consagrar a los dioses-y el delito de traición, de violación de la lealtad a Roma y al emperador se vinculaba al viejo derecho religioso que declaraba sacer a aquello que se había salido del mundo regulado por los dioses.

Por ello, la traición además de un delito, era una impiedad contra los dioses y la nemesis divina alcanzaba al traidor condenándolo a muerte, lo que permitía que los desertores y los traidores, aunque gozaran de la ciudadanía romana y tuvieran el rango social más elevado, fueran ejecutados mediante la crucifixión.

\section{LA RELIGIÓN OFICIAL}

\section{Los cultos tradicionales}

Los militares romanos destinados en Hispania, como todos los del Imperio, tenían como propio el culto a los viejos dioses cívicos tradicionales de Roma. Con esta premisa es normal que el dios que recibió más culto fuera luppiter, en su advocación de luppiter Optimus Maximus o dios supremo y supremo protector de Roma ${ }^{10}$.

\footnotetext{
7 J. Helgeland: «Roman Army Religion...», pp. 1.500-1.501.

8 Las ediciones del Feriale son numerosas. J. HelgeLAND: «Roman Army Religion...», pp. 1.481-1.484, proporciona una edición actualizada junto con una traducción inglesa.

9 J. HelgelAND: «Roman Army Religion...", pp. 1478-1480. Y. LE BoHEc: L'Armée..., p. 255.

10 E. BIRLEY: «The Religion of the Roman Army...», pp. 1.509-1.511.
} 
Esta divinidad era especialmente querida y familiar para los soldados, ya que el culto propiamente militar a las «insignias» estaba directamente relacionado con el de Júpiter, porque aquilae, signa y vexilla eran otras tantas manifestaciones del dios tutelar de Roma ${ }^{11}$. Además, la relación entre el culto a Júpiter y el culto imperial era evidente para los soldados, ya que en las ceremonias religiosas oficiales realizadas en los campamentos se entremezclaban estos dos cultos, además del culto a los signa.

Por ello, los soldados tendian a honrar a esta divinidad siempre que terminaban la realización de una misión y casi en cualquier circunstancia. Es posible considerar que estas manifestaciones de piedad que vinculaban los cultos a Júpiter, a las insignias y a los emperadores, además de una función religiosa clara, fuera también una manifestación de lealtad al ejército, al emperador, a Roma y a sus dios tutelar, y como tal una reafirmación de aquello que se juraba en el sacramentum.

Las dedicaciones de militares con guarnición en Hispania a Júpiter fueron erigidas durante todo el Alto Imperio, concentrándose especialmente en la segunda mitad del siglo $\|$ y bajo la dinastía Severa ${ }^{12}$. Las inscripciones al Padre de los dioses en la Península fueron consagradas por soldados y oficiales que prestaron servicio en las legiones ${ }^{13}$, y por tanto ciudadanos romanos, y también por algunos indígenas encuadrados en los auxilia ${ }^{14}$.

Algunas inscripciones, concretamente las que proceden de la zona minera de la provincia de León ${ }^{15}$, fueron dedicadas conjuntamente por soldados de la Legio VII Gemina y por hombres de algunas de sus unidades auxiliares, el Ala II Flavia, la Cohors I Celtiberorum y la Cohors I Gallorum. Estas dedicatorias son muy importantes, ya que no es normal que legionarios y auxiliares erijan conjuntamente inscripciones a los dioses. Creemos que Júpiter,

J. Helgeland: «Roman Army Religion...», pp. 1.475-1.476.

12 M. H. Gallego Franco: «Hispania y Ponnonia: Dos Modelos de Difusión del Culto en la Tríada Capitolina". Hart. XX, 1885, p. 181, considera que el culto a Júpiter se difunde en Hispania a través de una política deliberada del gobierno imperial, uno de cuyos agentes sería el ejército. Ya hemos afirmado que el Imperio conoció, fuera del ejército, la religión oficial, por lo que fue tal política sobre los no romanos no deja de ser una entelequia. Además, los soldados tenían obligación de creer en la religión tradicional, pero no tenían porqué hacer proselitismo.

13 Vide catálogo Tituli lovi Sacrum, números $1,2,3,4,5,6,7,8,9,10,11,12$, ¿13?, ¿14?, 15, 17, 19, 21, 22, 23, 24, 25, 26, 27, 28.

14 Vide catálogo Tituli lovi sacrum, números $3,4,5,6,7,8,9,10,11,12$, ¿13?, ¿14?, 16, 18.

15 Vide catálogo números 3-12. 
los signa y los emperadores fueron honrados conjuntamente por el estrecho contacto que legionarios y auxiliares mantenían en éste coto minero.

Sólo dos inscripciones fueron dedicadas a otras advocaciones del dios del cielo. Por un lado, un ara procedente de Clunia fue erigida en el año 6869 por un soldado de la Legio X Gemina a luppiter Augustas Vitor ${ }^{16}$, una advocación de Júpiter como vengador, especialmente querida por Galba, proclamado emperador en esta localidad. La otra advocación es la de luppiter Optimus Maximus Depulsor ${ }^{17}$ o protector, un epíteto de uso corriente para esta divinidad, aunque es posible que la inscripción pueda leerse, dada la abreviatura, como dedicada al Júpiter oriental o Dolichenus ${ }^{18}$.

Por el momento, no se conserva en Hispania ninguna inscripción erigida por un militar en honor de toda la Tríada Capitolina, y sólo tres epígrafes procedentes de Legio ${ }^{19}$, Tarraco y Segisamo fueron consagrados uno en favor de luno y Minerva ${ }^{20}$, otro en honor de luppiter, luno y Minerva ${ }^{21}$, y el otro a luno, luppiter Optimus Maximus y al Genius Stationis Segisamonensium ${ }^{22}$. A ellas se añade otra inscripción procedente de San Luron la mayor consagrada en honor de luno Regina ${ }^{23}$.

El resto del panteón también está poco representado, ya que Mars, el dios de la guerra, sólo aparece en cuatro epígrafes, tres de ellos dedicados por ciudadanos romanos ${ }^{24}$. Es de destacar que en sendos casos aparece acompañado de los epítetos Sancto ${ }^{25}$ y Pater ${ }^{26}$, este último relativamente extraño, indicando, tal vez, al Marte latino de la agricultura y no al guerrero.

16 Vide catálogo Tituli lovi Sacrum, $\mathrm{n} .^{\circ} 22$.

17 Vide Catálogo, Tituli lovi Sacrum, n. ${ }^{\circ} 2$.

18 Cfr. S. Perea Yébenes: «Las Manos de Júpiter Dolicheno: un Nuevo ejemplar de Riotinto (Huelva)». HAnt. XIX, 1995, pp. 226-227 y foto 9.

19 P. LE ROux: L'Armée..., p. 280 habla de la existencia de un santuario a estas divinidades en Legio. Ciertamente, en el aedes del princigia de todos los campamentos se rendía culto a las deidades principales del panteón romano, pero no por ello tenía que haber un Capitolium.

20 Vide catálogo. Tituli lunoni et Minervae Sacrum, $\mathrm{n} .{ }^{\circ} 1$.

${ }_{21}$ Vide catálogo. Tituli lunoni et Minervae Sacrum, $n .^{\circ}$ 2. En Tarraco, capital de la provincia Citerior y ciudad privilegiada con el status de Colonia Civium Romanorum es posible que si que existiera un Capitolium.

22 Vide catálogo, Tituli lunoni et Minervae Sacrum, $n .{ }^{\circ} 3$.

23 Vide catálogo, Tituli lunoni et Minervae Sacrum, n. 4.

24 Vide catálogo, Tituli Marti Sacrum, números 1, 3, 4.

25 Vide catálogo, Tituli Marti Sacrum, $n .{ }^{\circ} 1$.

26 Vide catálogo, Tituli Marti Sacrum, n. ${ }^{\circ} 4$. 
Diana ${ }^{27}$, la diosa de la caza, sólo fue honrada cuatro veces, una de ellas dudosa, aunque la primera, un ara votiva con una muy florida dedicación en un excelente latín, como corresponde a un culto legado senatorial, describe bastante bien como era el aspecto natural del territorium de la Legio VII Gemina a mediados del siglo $\|$, y además fue erigida como acción de gracias por un cazador empedernido ${ }^{28}$.

Las otras dos inscripciones proceden del campamento del Ala // Flauria en Petravinium (Rosinos de Vidriales, Zamora) y nuevas erigidas por sendos Praefecti de la unidad en los siglos $I \mathrm{y}$ III, respectivamente. Uno de ellos, T. Iunisus Quodretus, también era devoto de Marte ${ }^{29 "}$.

Otros dioses del panteón, como Mercurius ${ }^{30}$, Liber Pater ${ }^{31} 0$ Hércules ${ }^{32}$ recibieron aún menos culto por parte de los soldados de Hispania. Así mismo, la divinidad Sol Invictus, propia de la teología solar imperial del siglo $11{ }^{33}$, sólo tiene un adorador militar constatado en Hispania $^{34}$.

\section{Los cultos propiamente militares}

Los soldados romanos de guarnición en Hispania, al igual que sus camaradas del resto del Imperio, rindieron culto a las insignias militares, aquilae, signa y vexilla, que interpretaban como objetos sagrados relacionados con la divinidad suprema del panteón, Júpiter.

Los signa eran sagrados por sí mismos, de forma que tanto en marcha como en el campamento santificaban el lugar en el que se encontraban ${ }^{35}$. En los campamentos, mientras la unidad estaba acuartelada, los signa estaban depositados en el aedes, una capilla cuadrangular situada en el principia o edificio administrativo, en el que se encontraba el estado mayor de la unidad ${ }^{36}$.

\footnotetext{
27 Vide catálogo, Tituli Dianae Sacrum, números 1, 2.

28 Vide catálogo, Tituli Dianae Sacrum, números 3 y 4.

Vide catálogo, Tituli Marti Sacrum, n. 1.

Vide catálogo Tituli Aliis Diis Sacrum, n. 1.

Vide catálogo Tituli Aliis Diis Sacrum, n. ${ }^{\circ} 5$.

Vide catálogo Tituli Aliis Diis Sacrum, $\mathrm{n} .{ }^{\circ} 2$.

E. B|RLEY: "The Religion of the Roman Army...», pp. 1.518-1.519.

Vide catálogo Tituli Aliis Diis Sacrum, $\mathrm{n} .^{\circ} 5$.

J. HELGELAND: «Roman Army Religion...», p. 1.479.

J. HELGELAND: «Roman Army Religion...», pp. 1.476-1.477.
} 
Este aedes era el lugar más sagrado del campamento, ya que en él, además de depositarse los signa, se encontraban las imagines imperiales y las estatuas de los dioses principales, en especial la de luppiter. Tan sagrado era el aedes, que allí se encontraba la caja fuerte de la funidad ${ }^{37}$, en la que los soldados depositaban obligatoriamente una parte de su salario, que entregaban a la custodia de los portaestandartes de sus unidades, o Signiferes ${ }^{38}$.

En Hispania este culto se encuentra atestiguado por una serı de Inscripciones de la zona minera del oeste de la provincia de León ${ }^{39}$ y en otra procedente de Emporiae ${ }^{40}$, dedicadas en los aniversarios de la entrega del aquila de la Legio VII Gemina, del signum de la Cohors I Celtiberorum y del Aprunculorum de la Cohors I Gallica en honor de luppiter Optimus Maximus y por la salud de los emperadores reinantes.

Estas inscripciones relacionan todas las enseñas militares con el dios supremo romano, con Júpiter ${ }^{31}$, algo que era evidente para el caso de las aquilae de las legiones, pero que ahora se demuestra para el resto de las insignias. También aparece muy claro que este culto militar está relacionado con el culto imperial, gracias a la dedicación pro salute.

También es un culto militar, inscrito perfectamente dentro del conjunto de las religiones del Imperio, rendir homenaje a los genii de los lugares relacionados con el ejército ${ }^{42}$. En este marco se inscriben dos aras procedentes del campamento de Legio dedicadas por altos oficiales de la Legio VII Gemina al genius de la unidad ${ }^{43}$, así como otra procedente de la zona norte del conventus Bracaraugustanus, dedicada nada menos que al Genius Populi Romani ${ }^{44}$, también por un alto oficial de la Legio VII Gemina.

Además de estas tres dedicaciones tan explícitas, dentro del culto a los genii encaja también otro epígrafe procedente de Asturica Augusta, dedicado a finales del siglo "por un Dux de la Legio VII Gemina a varios

${ }^{37}$ G.R. Watson: The Roman Imperial Soldier..., p. 131. J. Helgeland: «Roman Army Religion..., p. 1.478.

38 Y. LE BOHEC: L'Armée... , pp. 51, 53, 63 y 233.

39 Vide catálogo Tituli Signae Sacrum: Aquilae, números 1-5, y Signa, números 1-5.

40 Vide catálogo, Tituli Signae Sacrum: Aquilae, $n .^{\circ} 6$.

41 Tertuliano. Apolog. I, 12. J. HeLgeLAND: «Roman Army Religion...», pp. 1.475-1.476.

42 J. HeLGeland: «Roman Army Religion...», pp. 1.499-1.500.

43 M.P. SPEIDEL: «The Cult of Genii in the Roman Army: on New Military Deity». ANRW. II, 16.2, 1978, pp. 1.544-1.548. Vide Catálogo. Tituli Genii Sacrum, números 2, 3.

44 M.P. Speidel: «The Cult of Genii...», p. 1543. Vide Catálogo. Tituli Genii Sacrum, $n{ }^{\circ} 6$. 
dioses y al genius praetorii de la unidad ${ }^{45}$, lugar donde residía el Legatus Legionis, y también el $d u x$, y se encontraba depositado el símbolo de su autoridad, y otro titulus dedicado al Genius stationibus Segisamonensium $^{46}$ en la misma época.

Otra inscripción, probablemente falsa, fue dedicada al genius de los campamentos ${ }^{47}$.

\section{El culto imperial}

Los soldados, según indica era el Feriale Duranum, tenian obligación de rendir culto a los divi o emperadores divinizados y además debían un respeto casi religioso al emperador reinante, al que juraban fidelidad.

En este marco se inscriben las relativamente abundantes dedicatorias erigidas a Júpiter por legionarios y soldados de unidades auxiliares en honor de algunos emperadores ${ }^{48}$, como Antonino Pío, Lucio Vero y Marco Aurelio, Cómodo, Septimio Severo, Julia Mammea, Severo Alejandro, Decio y Volusiano, lo que marca el auge de este culto entre los soldados de Hispania desde mediados del siglo II a mediados del III.

Con este culto también se relaciona el fragmento de brazo de bronce de una gran estatua de Septimio Severo procedente del campamento de Petavonium ${ }^{49}$.

Como ya hemos afirmado, hay bastantes inscripciones que relacionan el culto imperial con el culto a Júpiter ${ }^{50}$ y con el culto a las insignias militares.

45 M.P. SPEIDEL: «The Cult of Genii...», pp. 1.549-1.550. Vide catálogo, Tituli Genii Sacrum, n. ${ }^{\circ} 4$.

46 Cfr. M.P. SPeIDEl: "The Cult of Genii...», pp. 1.549-1.550. Vide Catálogo Tituli Genii Sacrum, n. ${ }^{\circ} 8$.

47 Vide catálogo, Tituli Genii Sacrum, n. ${ }^{\circ} 5$.

48 Vide catálogo Tituli Pro Salute Imperatoris Sacrum, números 1-18.

49 R. MARtín Valls y G. Delibes DE CAStro: «Hallazgos arqueológicos en la Provincia de Zamora IX». BSAA XLVIII, 1982, pp. 54-61.

50 En la Lex Irnitana cap. 69 -...quisque corum (decurionum) iuret per lovem et divum". Aug(ustum) et divum Claudiuom et divum. Vesp(asianum) Aug(ustum) et divum Titum Augustum et Genium Imp(eratoris) Domitiani Aug(usti) deosque penates...-, en época de Domiciano se obligaba a los decuriones del municipio a jurar por Júpiter, los emperadores divinizados y por el genius del propio Domiciano. La intención que se aprecía aquí es la de asimilar el culto a los Divi al de Júpiter, y también la de equiparar al propio Domiciano con esos Divi y con Júpiter. Si esto ocurría en el mundo civil, en el mundo militar está equiparación debía ser aún mayor, y en este contexto debemos encajar las dedicaciones pro salute Imperatoris. 


\section{LAS DEVOCIONES PRIVADAS DE LOS SOLDADOS}

Los soldados, además de ser devotos de la religión oficial del ejército, podían creer en aquello que más les gustase y no fuese contrario a las leyes y usos del Imperio. La única limitación es que estos cultos no podían practicarse en el interior de los campamentos.

\section{Las abstracciones divinizadas y las deidades latinas menores}

En general, los soldados de Hispania, como todos los habitantes del Imperio, eran bastante supersticiosos, y así rendían culto a algunas abstracciones y divinidades menores como eran Fortuna ${ }^{51}$, el Numen ${ }^{52}$, los genii de algunos lugares - concretamente los de los conventi Asturecensis ${ }^{53}$ y Bracaraugustanorum- ${ }^{54}$, la Victoria ${ }^{55}$, las Nimphae ${ }^{56} \mathrm{o}$ los Lares Viales ${ }^{57}$.

En el caso de la Victoria, la única inscripción conservada permite pensar que esta dedicada más a la Victoria indígena que a la romana, ya que fue erigida por un soldado de origen céltico que al tiempo dedicó otro epígrafe a la deidad lusitana Terabrura, que podría ser la Victoria.

Respecto a las Nimphae, también es posible que algunas dedicatorias fueran realizadas a divinidades indígenas de las aguas asimiladas a las ninfas latinas.

Dentro de este capítulo, también es posible incluir a los dioses manes, que aparecen en las numerosas inscripciones funerarias de soldados de los siglos II-III encontradas en la Península. Tras cotejar todo el elenco de epitafios de militares conservados de estos dos siglos, parece claro que la colocación de esta dedicatoria es optativa ${ }^{58}$, por lo que la deducción lógica es que sólo la inscribían los que creían en la existencia de los manes de los antepasados.

\footnotetext{
Vide catálogo, Tituli Fortunae Sacrum, números 1-4.

Vide catálogo, Tituli Numini Sacrum, números 1, 2.

Vide catálogo, Tituli Geniis Sacrum, n. ${ }^{\circ} 1$.

Vide catálogo, Tituli Geniis Sacrum, n. ${ }^{\circ} 7$.

Vide catálogo, Tituli Aliis Diis Sacrum, n. ${ }^{\circ} 3$.

Vide catálogo, Tituli Nimphis Sacrum, números 1-4.

Vide catálogo, Tituli Aliis Diis Sacrum, $\mathrm{n}^{\circ} 4$.

Cfr. catálogo, de P. LE Roux: L'Armée..., pp. 173-252.
} 
Otra abstracción que recibió culto fue Nemesis (La Venganza divina), que fue honrada con un ara en Petavonium ${ }^{59}$, donde también se documenta una placa votiva de mármol con impresiones de pies ${ }^{60}$, algo característico de este culto.

\section{Los cultos orientales}

Los soldados rindieron también culto a divinidades orientales salvadoras, aunque en Hispania poseemos un número muy pequeño de testimonios.

Uno de ellos fue dedicado en honor de Mithra ${ }^{61}$ por un frumentarias en el mitreo de la Colonia Emerita Augusta. Este soldado posiblemente se convirtió en devoto de este dios salvador de origen iranio a lo largo de sus viajes como espía imperial ${ }^{62}$.

Otra inscripción ${ }^{63}$, procedente del campamento de Legio, casi seguro que del valetudinarium, único lugar de los campamentos en el que era posible erigir inscripciones a otros dioses distintos de los oficiales, fue dedicada a los dioses sanadores de origen griego Aesculapius y Salus, junto con Serapis e Isis, divinidades de origen egipcio que prometían a sus fieles la salud, la felicidad y la vida en el más allá, por lo que también podrían considerarse sanadores.

\section{El culto a divinidades indígenas de Hispania}

Las deidades de los pueblos célticos de Hispania también fueron honradas por los soldados de las unidades del ejército romano destacadas en la Península.

59 Vide catálogo, Aliis Diis Sacrum, $\mathrm{n} .^{\circ} 5$.

60 R. Martín Vals, M. V. Romero Carnicero y S. Carretero Vaquero: «Aras votivas de Petavonium". Zephyrus, XLVIII, 1995, pp. 334-336.

61 Vide catálogo, Tituli Diis Orientalis Sacrum, $n .{ }^{\circ} 2$.

62 La opinión tradicional sobre la difusión del Mithraismo en Hispania indica que fue realizado por militares (Cf. M. A. DE Francisco CASADO: El culto de Mithra en Hispania, Granada, 1989, pp. 67-68, seguida por M. R. García MARTínez: “El Culto a Mithra en Hispania y Germania. Estudio Comparativo". Hart. XX, 1996, pp. 204-205), lo que a la vista de la documentación es falso.

63 Vide catálogo, Tituli Diis Orientalis Sacrum, n. ${ }^{1 .}$ 
Ninguna recibió más de dos o tres inscripciones en su honor, y desconocemos cuales eran los atributos de estas divinidades, lo que es común con el resto de las deidades originarias de Hispania. La primera excepción posible, ya indicada, es la de la diosa Trebaruna, que tal vez sea equivalente a la Victoria latina, ya que en Egitania un soldado indígena levanto sendas aras votivas a las dos divinidades ${ }^{64}$, por lo que es probable que sean dos formas, una indígena y otra latina, de la misma abstracción divinizada.

La otra excepción viene marcada por la Larocuus ${ }^{65}$, una divinidad relacionada con la sierra Larouca, que fue sincretizada con el luppiter latino para poderle erigir una inscripción, por lo que sus atributos no debían diferir demasiado de los del padre de los dioses ${ }^{66}$.

Las tres inscripciones dedicadas a divinidades con el radical Band- ${ }^{67}$ parece que fueron dedicados a deidades asimiladas a la Tutela ${ }^{68}$. Por otro lado, la diosa Cabarcenséis ${ }^{69}$ estaba asimilada a Minerva ${ }^{70}$ y Turiacus ${ }^{71}$ era una divinidad de las aquas ${ }^{72}$.

La única divinidad excepcional que aparece en este elenco sería la diosa lunar en cuyo honor un soldado de Mago (Mahón, Menorca) levantó un ara ${ }^{73}$, ya que esta deidad no es indígena sino que tiene origen púnico.

La mayoría de estas inscripciones fueron dedicadas por soldados que prestaron servicio en las legiones romanas destacadas en Hispania, sobre todo en la Legio VII Gemina. Esta veneración privada, siempre fuera del campamento, a estas divinidades puede estar relacionada con la progresiva extensión de la ciudadanía romana a los indígenas naturales de la Península, en especial a los de la zona noroeste, que adquirieron está categoría y pudieron tomar un nombre romano y alistarse en una Legión, pero no renunciaron a sus dioses privados.

\footnotetext{
Vide catálogo, Tituli Diis Populorum Hispaniae Sacrum, n. ${ }^{\circ}$ 8. y Tituli Alteris Diis Sacrum, n. ${ }^{\circ} 3$. Vide catálogo, Tituli Diis Populorum Hispaniae Sacrum, $\mathrm{n} .^{\circ} 5$.

J. M. ${ }^{a}$ BLÁZQUeZ: Diccionario de las Religiones Primitivas de Hispania. Madrid 1975, p. 117.

Vide catálogo, Tituli Diis Populorum Hispaniae Sacrum, números 2, 3, 4.

J.M. ${ }^{\text {a }}$ BLÁZQUEZ: Diccionario..., p. 43.

Vide catálogo, Tituli Diis Populorum Hispaniae Sacrum, n. ${ }^{\circ} 10$.

J. M. ํ BLÁzQUEZ: Diccionario..., p. 51, siguiendo a CIL X, 1301 y 1306.

Vide catálogo, Tituli Diis Populorum Hispaniae Sacrum, n. 7 .

J. M. ${ }^{a}$ BLÁZQUEZ: Diccionario..., pp. 176-177.

Vide catálogo, Tituli Diis Populorum Hispaniae Sacrum, n. 11.
} 


\section{CONCLUSIONES}

La religión practicada por los soldados romanos destinados en Hispania durante el Alto Imperio está fuertemente teñida de oficialidad, ya que la mayoría de las inscripciones y testimonios religiosos que han llegado hasta nosotros están referidos a los cultos a Júpiter Óptimo Máximo, a otras divinidades del panteón tradicional, al culto a las insignias militares y al culto imperial.

Por contra, las devociones privadas de los soldados son pocas y con testimonios bastante reducidos, hasta el punto que podemos afirmar que el culto a divinidades indígenas gozó de mayor popularidad que el culto a dioses orientales. Por ello consideramos que a la hora de explicar la difusión de los cultos orientales en Hispania, hay que pensar más en comerciantes que en soldados.

Esto es normal si se piensa que las unidades de guarnición en Hispania durante los siglos II al IV - Legio VII Gemina, Ala II Flavia, Cohors I Celtiberorum, Cohors I Gallica, Cohors II Gallica y Cohors III Lucensium - apenas salieron de la Península y se nutrieron fundamentalmente con levas de origen hispano. Los soldados al alistarse llevaban consigo sus religiones particulares, entre las que difícilmente podian encontrase divinidades orientales, ya que la mayor parte de los reclutas procedía de las zonas del asentamiento de las unidades, el Noroeste de Hispania, la zona menos abierta al resto del mundo romano. 


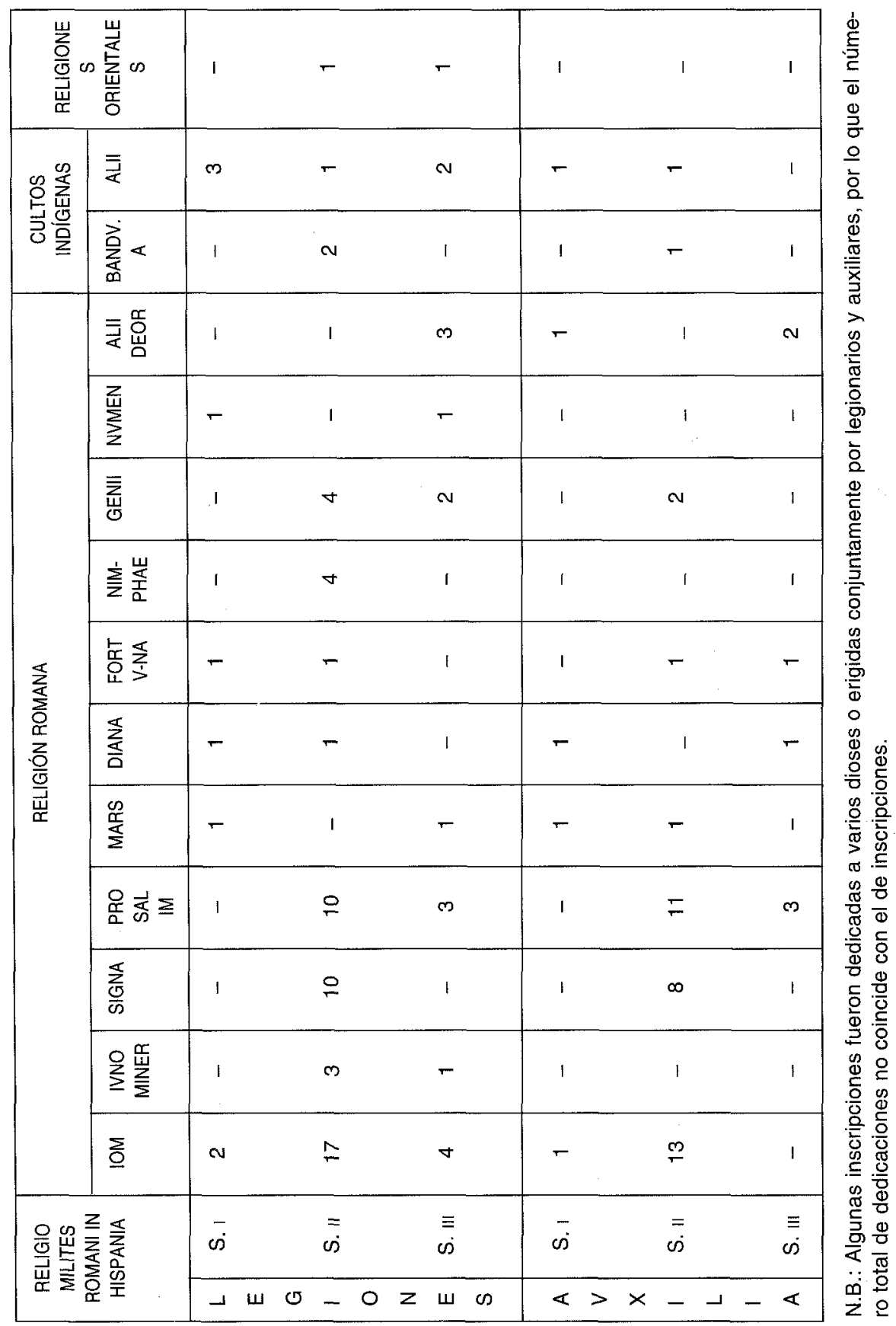




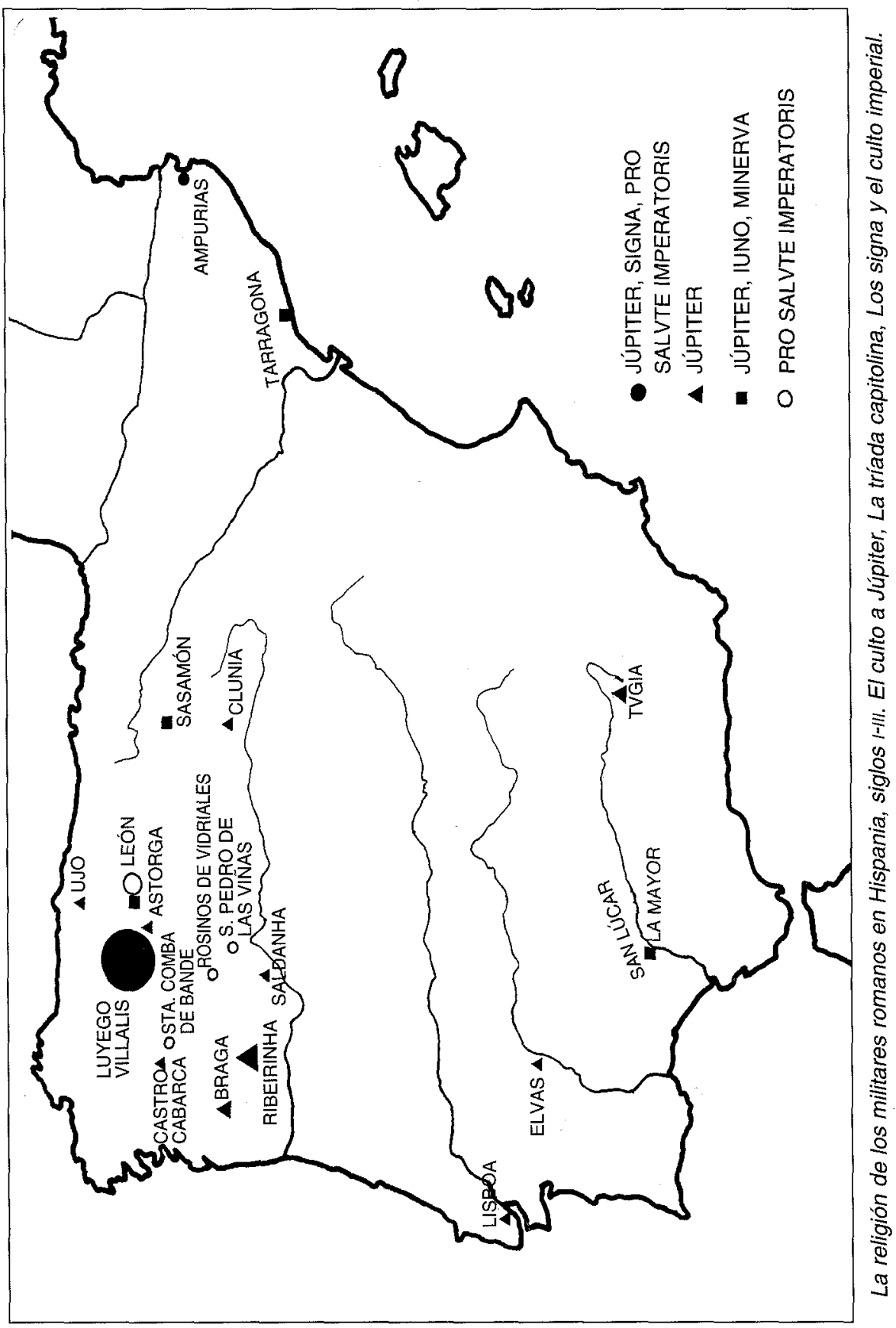




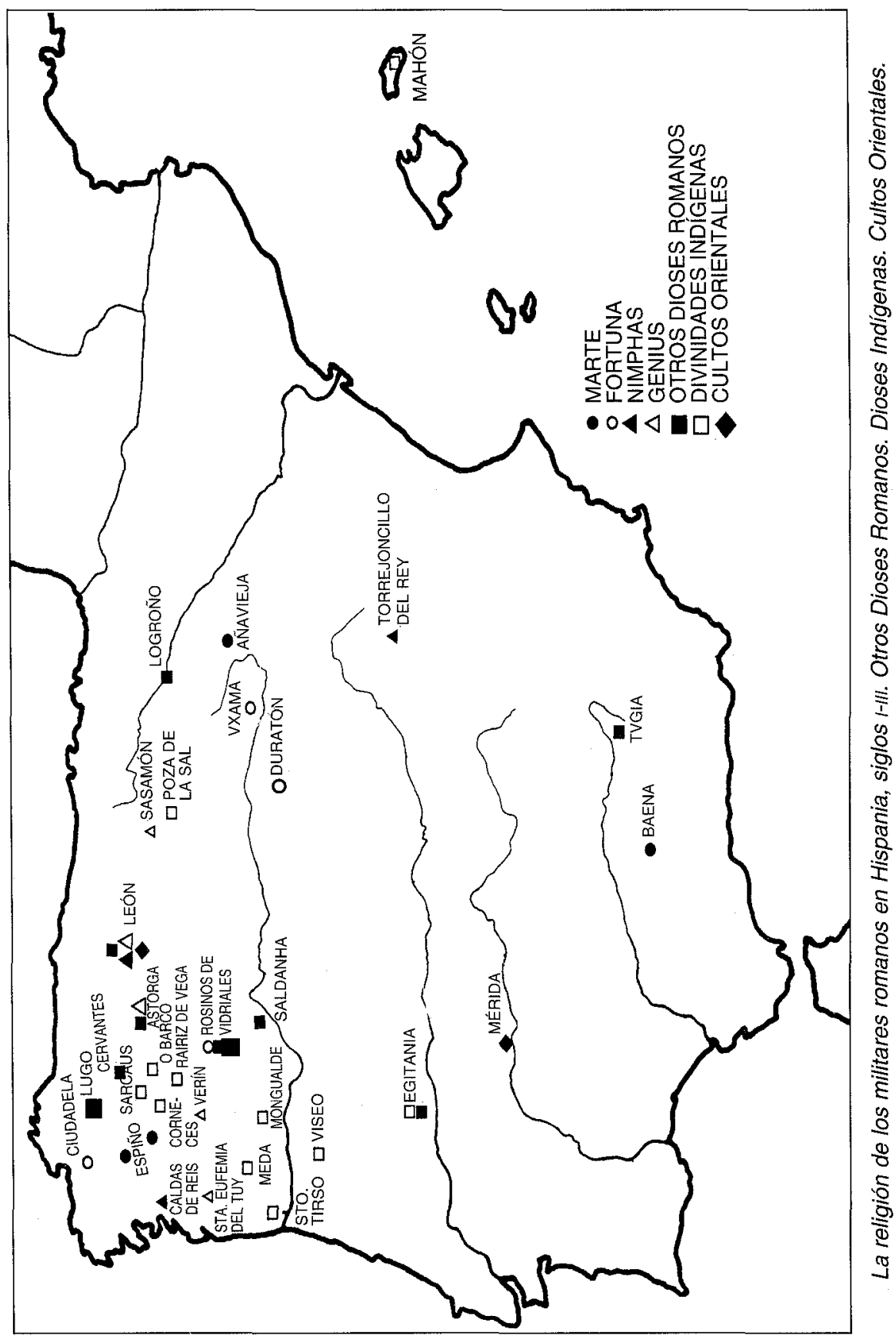


CATÁLOGO DE INSCRIPCIONES VOTIVAS HISPANAS DEDICADAS POR MILITARES

\section{TITVLI IOVI SACRVM}

1. Ujo (Asturias). C/L II, 5733. 2.․ 1/2 S. II.

I (ovi) - O(ptimo) · M(aximo)/ LVCIVS/ CORONA - S/EVERVS M/ILES LEG(ionis) VII/ GEM $<I N>A E$ ) (centuriae) VE/TTI ET OCTAIVIA PROCV$L A / V$ (otum) S(olvit) L(ibens) M(erito).

2. Saldanha (Miranda do Douro, Portugal). AE. 1974, 393bis = P. LE ROUX. L'Armée Romaine des Provinces Ibérigues d'Aauste a l'Invasion de 406. París, 1982, n. ${ }^{\circ}$ 200. S. III.

I (ovi) - O(ptimo) - M(aximo) $\cdot D$ (epulsor vel Dolicheno ?)/ DOMITVS/ PEREGRINVS/VET(eranus) LEG(ionis) VII/ GE(minae) P(iae) F(idelis)/ $V$ (otum) S(olvit) L(ibens) M(erito).

3. Luyego de Somoza (León). ILS $5945=\mathrm{T}$. Mañanes. Epigrafía y Numismática de Astorga Romana y su Entorno. Salamanca, 1982, n. ${ }^{\circ} 112$. 11-junio-Int. 138-161.

[I (ovi) - O(ptimo) - M(aximo)/ PRO SALVTE/T(iti) AEL(ii) · HADRIANI/ ANTON(ini) [hedera] AVG(usti) [hedera] PB/ VEX(illatio). LEG(ionis) .VII. $G$ (eminae) $\cdot F($ elicis)/ SVB CVR(a) . IVL(ii) [hedera] MAR/CI(i) $\cdot C$ (enturio legionis) - EIVSD(em) [hedera] ET . VAL(erii) VICTORINIS DEC(urionis) OB/ DIEM NATA(lis) · AQVILAE/V(otum) · S(olvit) · L(ibens) · M(erito).

4. Villalís (León). T. Mañanes, $n .{ }^{\circ}$ 117. E. Schallmayer et alii. Der Römische Weibebizirk von Osterburken I: Corpus der Griechischen und Lateinischen Benefiziar Inscriften des Romischen Reiches. Stuttgart, 1990, n. ${ }^{\circ} 858.22$-abril-175.

I (ovi) - O(ptimo) - M(aximo) - S(acrum)/ PRO SALVTE · IMP(eratoris)/ CAES(aris) · M(arci) · AVR(elii) . ANTIOJ/NINI · AVG(usti) · OB . NAT[AL(em)]/ APRVCVLORVM/ MILITES - CO[H(ortis) · I GA[L(lorum)]/ SVB CVRA - M(arcii) - SENTI(i)/ BVCCONIC(i) - )(centurionis). COH(ortis) . EIVS[D(em)]/ ET - VAL(erii) - SEMPRONIA[NI]/ B(ene)F(iciarii) PROC(uratoris) $\cdot A V G[($ usti) $] / \cdot X \cdot K($ alendas $) \cdot M A I(a s) \cdot P I S O N E ~ E T \cdot I V L I A N O$ $C O(n) S($ ulibus).

5. Villalís (León). T. Mañanes, $n .{ }^{\circ} 114$. E. Schallmayer et alii. $B l, n .^{\circ}$ 857. 11-junio-163. 
I (ovi) - O(ptimo) - M(aximo)/[P]RO SALVTE . M(arci) - AVRELI(i) .

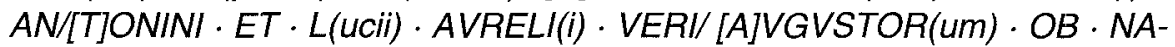
TALE $(m) \cdot A Q V I I] / L A E \cdot V E X I L L A T I O$ LEG(ionis) · VII - G(eminae) F(elicis)/ SVB - CVRA. LICINI(i) . PATERNIIII) · (centurionis) · LEG(ionis) · EIVSD(em) - ET · HERMETIS/ AVGVSTOR(um) - LIB(erti) - PROC(uratorum) - ET - LV/CRETI(i) - PATERNI - DEC(urionis) COH(ortis)/ I - CELT(iberorum) - ET . FABI(i) MARCIANIIJ/ B(ene)F(iciarii) PROC(uratoris) - AVGVSTOR(um) · ET . IVLI(i) - IVLANI SIGN(iferi) · LEG(ionis)/ EIVSD(em) · III - ID(ibus) - IVNIAS/ LAELIANO ET PASTORE CO(n)S(ulibus).

6. Castro de San Cristóbal, Villalís (León). F. Diego Santos. EPRLe. León 1989, n. ${ }^{\circ} 38$. ¿163?

I (ovi) - O(ptimo) · M(aximo)/ PRO SALVTE M(arci) AVRELI(i)/ ANTONINI ET L(Ucii) AVRELI(i) VERI/ AVGVSTORVM OB NATALE(m)/ [APRVNCVLORVM] MILITES/ COH(ortis) I GALL[O]R(um)/ SVB CVRA [H]E[R]MET(is) AVG[VSTOR(um) LIB(erti)]/ [P]R[OC(uratoris)] ET LVCRETIII] PATERN[I]/ [DEC(urionis)] COH(ortis) [I CELTIB(erorum)] ET FVLIVI(i)- . . . .

7. Villalís (León). $C / L \mathrm{II}, 2556=\mathrm{T}$. Mañanes, $n .{ }^{\circ} 115$. E. Schallmayer et alii. $B I, n .{ }^{\circ} 855.22$-abril-163.

I (ovi) · [O(ptimo) - M(aximo)/ PRO SALVTE M(arci) AVRELI(i)]/ ANTONIINI ET L(UCii) AVRELI(i)] VERI AVGIVSTORVM OJB N[ATALE(m)]I APRVNCV[LOR(um)] MIL[ITES]/ COH(ortis) I GALL(orum) SVB CVRA [ZO/I]LI AVGVSTOR(um) LIB(erti) · PROIC(uratoris)/ ET VAL(erii). FLAVI(i)) (centurionis) $\mathrm{COH}$ (ortis) - EIIVS/DE]M - ET VAL(erii) VALENTIS [B(ene) F(iciarii)/ PRIOC(uratoris) AVGVSTIOR(um)] ET IV[LI(i)/ IVL]IANI SIGNIFER(i) LEG(ionis) VII G(eminae) [F(elicis)/ X K(alendas)] MAIAS PVD(ente) [ET FVF(idi ?) CO(n)S(ulibus)].

8. Villalís (León). CIL II, $2553=\mathrm{T}$. Mañanes, n. ${ }^{\circ} 116$. E. Schallmayer et alii. $B I, n .^{\circ} 856.13$-octubre-167.

I (ovi) - O(ptimo) - M(aximo)/ PRO SALVTE M(arci) - AVRELI(i) ANTONINI/ ET · L(ucii) · AVRELI(i) - VERI AVGVSTORVM/ OB NATALE(m) SIGNOR(um) - VEXILLATIO/ CIO]H(ortis) I CELTIB(erorum) SVB CVRA ZOILI/ AVGVSTOR (um) - LIB(erti) - PROC(uratoris) - ET . VAL(erii) - FLAVII(i)]/) (centurionis) · COH(ortis) I GALL(orum) - ET AELI(i) FLAVI(i) B(ene). F(iciarii) PRIOC(uratoris)/ AVGVSTOR(um) . ET LVCRETI . MATER/NI . IMAG(iniferis) · LEG(onis) · VII G(eminse) F(elicis) · ET IVLI(i) SE/DVLI 
TESSERARI - C(ohortis) · I C C(eltiberorum) - POSITA IDIB(US) OCTOBRIB(us) IMP(eratori) - L(ucio) · AVRE/LIO VERO III ET QVADRATO $C O(n) S(u l i b u s)$.

9. Luyego de Somoza (León). T. Mañanes, n. ${ }^{\circ 113.11-j u n i o-181 .}$

I (ovi) - O(ptimo) - M(aximo)/ PRO SALVTE · L(ucii) - AVR(elii)/ COMMODI - AVG(usti) - OB/NATALE (m) $\cdot A Q V I L<A>E \cdot L E G($ ionis)/ VII G(eminae) - F(elicis) · SVB · CURA - M(arci)/ AVR(elii) - EVTYCHETIS - A(ugusti)/PROC(uratoris) ET · M(arci) MES(ii)/ [-]RIS ) (centurionis) $\mathrm{COH(ortis)}$ I GALL\{l\}(orum)/ET · AVITI . PATERN(i)/AVG(usto)/ET . BVR<R>O CO(n)S(ulibus).

10. Villalís (León). T. Mañanes. $\mathrm{n} \cdot{ }^{\circ} 118.12$-junio-184.

l(ovi) - O(ptimo) - M(aximo)/ PRO SALVTE M(arci) - A[VR(elii]/ ANT[ONINI AVG(Usti)]J OB NATALE(m) AQVI/LAE VEXILLA[TIO]/ LEG(ionis) VII G(eminae) F(elicis) SVB CV/RA . AVR(elii) - EVTYCH[IS]/ AVG(usti) LIB(erti) PROC(uratoris) - ET - VIAL(erii)/ SEMPRONIANI [DEC(urionis)]/ ALAE /I FLAVIAE IIII I[D(ibus)/IVN(io) - MARVLLIO - ET . AELIANO/ CO(n)S(ulibus).

11. Villalís (León). T. Mañanes, $\mathrm{n}^{\circ}$ 119. 22-abril-195.

I [(ovi) O(ptimo) M(aximo)/ PRO SALVTE . M(arci) AVRE/LI COMMODI AN]/TONINI PII - FEL(icis) AV[G(usti)]/ GER(manici) - MAX(imi) - TRIB(uniciae) - POT(estati)/ IMP(eratoris) XV CO(n)S(ulis) VI . OB - N[A]/TALE (m) . APRVCVLORVM/MIL(ites) COH(ortis) I . GAL(lorum) - SVB . CVIRA]/ AVRELI(i) - FIRMI - AVG(usti) - LIB(erti)/MET(allorum) - ET · VALERI(i) MAR$C E L L[I] / D E C$ (urionis) $\cdot A L($ ae) $\| \cdot F L$ (aviae) $\cdot X \cdot K$ (alendas) $\cdot M A[I / A] S$ OPILIO PEDONE ET/ [B]RADVA . MAVRIICO CO(n)S(ulibus)].

12. Castro de Corporales (León). T. Mañares, n. ${ }^{\circ}$ 148. 12-junio-163.

I (ovi) - O(ptimo) - M(aximo)/ [PR]O SALVITE M(arci) - AV]RELI(i) ANTONINI ET L(Ucii) AVRE/LI(i) - VERI AVGVSTOR(um) OB NATALE(m) $A<Q>V<I L>A E /$ VEXILLATIO LEG(ionis) VII <G(eminae) F(elicis) $>$ $S\{A\} V B$ CVRA LI/CENI(i) (sic) PATERNI) (centurionis legionis) EIVSD(em) <ET> HERMIITI(s) (sic) AVGVSTOR(um) PROCV(ratoris) ET LVCRI/TI(i) (sic) PATE $<R>N E$ (sic) DEC(urionis) COH(ortis) I CELT(iberorum) $E T$ AEMV(Iii) (sic)/ $A E C I A N(i) B(e n e) F$ (iciarii) $P<R>O C$ (uratoris) A $<V>G V T O R$ (um) ET AVLI(i) (sic)/ AVLIANI SIGN(iferi) LEG(ionis) EIVSD(em) IIII ID(ibus) I/[VNI]A(s) LAELIANO ET/ [PASTORE $C O(n) S$ (ulibus). 
13. Villalís (León). $A E .196566=$ T. Mañares, n. ${ }^{\circ}$ 120. Int. 161-180.

I (ovi) [hedera] O(ptimo) [hedera] M(aximo) [hedera]/ PRO SALVTE [hedera] M(arci) [hedera] AVRELI(i) [hedera] ANTONINI/ . . . . . . .

14. Luyego de Somoza (León). T. Mañanes, n. ${ }^{\circ} 145.2^{\mathrm{a}}$ 1/2 S. I. I (ovi) [O(ptimo) M(aximo)/ P]R[O SALVTE- - - - - . - ]/ M[ILITES- - -

15. Astorga (León). CIL II $2634=\mathrm{T}$. Mañares, $n .^{\circ}$ 12. Septimio Severo-Clodio Albino (193-197).

I (ovi) - O(ptimo) - M(aximo)/ SOLI INVICTO LIBERO/ PATRI GENIO PAETOR(ii)/ Q(uintus) - MAMIL(ius) . CAPITOLINVS/IVRID(icus) PER FLAMINIAM/ ET VMBRIAM ET PICENVM/ LEG(atus) . AVG(usti) PER. ASTVRIAM ET/ GALLAECIAM - DVX · LEG(ionis) - VII · [G(eminae)]. $P($ iae $) \cdot[F($ elicis) )] $/$ PRAEF(ectus) · AER(arii) - SAT(urni) - PRO - SALVTE/ SVA . ET . SVORVM.

16. Prozello, concelho de Ames, Braga. CIL II, 5610. 19 1/2. S. II.

$C$ (aius) · AEMIL(ius)/ VALENS/ EQ(ues) AL(ae) . FL(avise) . / TVRMA(e) [P]RI/MANI [IOIVI O(ptimo) M(aximo)/ V(otum) S(olvit) L(ibens) $M($ erito).

17. Ribeirinha, Toricella, Vila Pouça de Aguiar (Vila Real, Portugal). AE. $1980,582.1 . \stackrel{2}{1 / 2} \mathrm{~S}$. II.

$Q$ (intus) ANNIVS/MODESTVIS]/M(iles) L(egionis) VII G(eminae) $F$ (elicis)/ IOVI O(ptimo) MA(ximo).

18. Castro Cabarca, Castelo de Val (Orense). AE. 1981, 528. 14-70.

I (ulius) CAS(sius) . CAEN(us)/ TAMAC(ano) ) (castello) NEM(entensi)/ $D E C$ (urio) · AL(ae) - I · GIG(urrorum)/ COMP(luribus) BEL(lis) TORQ (uibus)/ PHAL(eris) BIS/ DONATVS/ IOVI O(ptimo) M(aximo) V(otum)/ L(ibens) M(erito).

19. Braga. FE, n. ${ }^{\circ} 44=A E .1993,196 . \mathrm{S}$. 11.

I (ovi) - O(ptimo) - M(aximo)/G(aius) - IVL(ius) - SATTVRNINVS/MIL(es). LE(gionis)/VII . GEM(inse)/F(elicis)/ AFER.

20. Ribeirinha, Fegresia de Tres Minas, Vila Pouça de Aguiar (Vila Real, Portugal). EE. VIII, 109. S. II.

I (ovi) - O(ptimo) - M(aximo)/MIL(ites) $C<O>H$ (ortis) · / GALLI/CAE EQ(uitatae)/C(ivium) $\cdot R$ (omanorum) $\cdot V($ otum $) \cdot S($ olverunt) $/ L$ (ibentes) $\cdot M($ erito). 
21. Ribeirinha, Fegresia de Três Minas, Vila Pouça de Aguiar (Vila Real, Portugal). CIL II, 2389. S. III.

I (ovi) O(ptimo) M(aximo)/ VOT(um) . SOL(verunt)/ MIL(ites) . LEG(ionis)/VII G(eminse) P(iae) CA/TVLLINVS P(osuit).

22. Clunia (Burgos). $A E, 1969-70,274=1980,147.69-70$

IOVI AVG(usti)/VLTORIS SACRVM/L(Ucius) VALERIVS PATERNVS/ MIL(es) LEG(ionis) X GEM(inae)/ OPTIO) (centuriae) CENSORIS EXS (sic)/ VOTO.

23. Lisboa (Portugal) $C / L$ II, $5099=\mathrm{P}$. Le Roux. L'Armée. n. ${ }^{\circ} 96 . \mathrm{S}$. I. IOVI OPT(imo)/ MAX(imo) C(aius)/ CASSIVS FV/NDANVS/ VETERANVS/ V(otum) S(olvit) L(aetatis) A(nimo) L(iens).

24. Juramenha, prope Elvas (Portugal). CIL II, $151=$ P. Le Roux. L'Armée. n. ${ }^{\circ} 101.2$. $1 / 2 \mathrm{~S}$. II.

[-] IVLIVS [- F(ilius)/ M]AXSVM[VS (sic)/ M]ILES [LEG(ionis) VII GEMIN(ae)/ F]ELICIS I(ovi) [O(ptimo) M(aximo]/ V(otum) S(olvit) L(ibens) $M($ erito).

25. Ampurias (Gerona). CIL II, $6183=I L S 2293=P$. Le Roux. L'Armée. n. ${ }^{\circ}$ 240. 10-junio-Int. 130-180.

I (ovi) O(ptimo) M(aximo)/VEXILLATIO/ [L]EG(ionis) VII G(eminae) F(elicis)/ [S]VB CVRA/ [I]VNI(i) VICTO/RIS) (centurionis) LEG(ionis EI/VSDEM OB NA/[TA]LEM · AQVILAE.

26. Tugia. $C / L$ II, $3327=\mathrm{P}$. Le Roux. L'Armée. n. ${ }^{\circ}$ 256. S. III.

NVMINI SAC(rum)/ I(ovi) O(ptimo) M(aximo)/ --- CARM - . - -/VOT(um) SOL(verunt) LI(bentes)/ MER(ito) VETERA(nus)/ LEG(ionis) VII GEMIN(ae)/PI $<A>E$ FE! ICIS.

27. Segisamo. Gerion, 10, 1992, pp. $259-273=$ CIL II, $2915=$ HEpig, 4, 1994, n. ${ }^{\circ} 201$. E. Schallmayer et alii. BI, n. ${ }^{\circ} 834$. Fin s. II.

[I (ovi) O(ptimo) M(aximo) - IVN(oni) REGIN]A[E/ ET GENIO] S/[T]ATIONIS - SEGI/SAMONENSIVM/ [-] AELIVS MARI/TIMVS B(ene)F (iciarius) - $C O(n) S$ (ularis)/ EXEDRAM · / CVM B(alnei?)/ D(e) S(ua) F(aciendum) $C$ (uravit).

28. Tarraco. RIT, 43 = P. Le Roux. L'Armée. n. ${ }^{\circ} 152.2^{\text {a }} 1 / 2$ s. II. E. Schallmayer et alii. BI. ${ }^{\circ}{ }^{\circ} 854$. Fin siglo II. 
[l (ovi) O(ptimo) M(aximo)/ IVNONI ET]/ MINER[VAE]/ L(ucius) VALERINS FLAVVS/ B(eneficiarius) M(iles) L(egionis) VII G(eminae) F(elicis)/ PRO SALVT(e) COL/LEGI(i) STRATO(rum)/V(otum) S(olvit) L(ibens) $M$ (erito).

\section{TITVLI IVNONI ET MINERVAE SACRVM}

1. León. $A E .1911,94.10$-junio-227.

In centro: MINERVAE ET IVNONI VICTRICI ACJ/ PATRIAE CONSERVIATRICI PRO SAL(ute) IVLIAE MAMMEAE] MATRII [I]MP(eratoris) - CAES(aris) · M(arci) · AVR[EL(ii)]/ [S]EVERI [ALEXANDRI] PII FEL(iciS)/ AIVG(USti) ET MATRIS SENATJVS ET CAST/ROR IVM ET PATRIAE- - -SJACRVM/ - - -

In latera: DEDIC(ato) III ID(ibus) IVN(ias)/ [ALBI]NO ET/ [MAXI]MO CO(n)S(ulibus).

2. Tarraco. RIT, 43 = P. Le Roux. L'Armée, n. ${ }^{\circ} 152$. 2. $^{\text {a }} 1 / 2 \mathrm{~s}$. II. E. Schallmayer et alii. $B I, \mathrm{n} .^{\circ} 854$. Fin siglo I. Vide $I O M, \mathrm{n} .^{\circ} 28$.

3. Segisamo. Gerion, 10, 1992, pp. 259-273 = CIL II, $2915=$ HEpig, 4, 1994, n. ${ }^{\circ} 201$. Vide $I O M, \mathrm{n} .{ }^{\circ} 27$.

4. San Zuron La Mayor. CIL II, 12-6-7. S. II.

IVNONI - REGINAE, M(arcus) - CALPVRIVIVS/ M(arci) - F(ilius) · GAL(eria) - Seneca - FabivS - TVRPIOI SENTIANVS. · PRIMVS - PILUS · LEGIONIS - PRIMAE - ADIVTRICIS/ PROCVRATOR - PROVINCIAE - LVSITANIAE/ET. VETTONIAE . PRAEFECTVS CLASSIS/ PRAETORIAE - RAVENNATIS · EXI ARGEN T(i) - LIBRIS - CENTVM - D(ono) - D(edicavit) ·

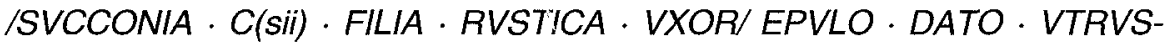
$Q(U e) \cdot S E X V S / D E D I C A T$.

\section{TITVLI SIGNAE SACRVM}

\section{AQVILAE}

1. Luyego de Somoza (León). ILS, $5945=\mathrm{T}$. Mañanes, $n .^{\circ} 112$. Vide IOM, n. 3 . 
2. Villalís (León). T. Mañanes, $n .^{\circ} 114$.

Vide $I O M$, n. $^{\circ} 5$.

3. Luyego de Somoza (León). T. Mañanes, n. ${ }^{\circ} 113$.

Vide $I O M, \mathrm{n} .{ }^{\circ} 9$.

4. Villalís (León). T. Mañanes, $n \cdot{ }^{\circ} 118$.

Vide IOM, n. 10.

5. Castro de Corporales (León). T. Mañanes, $n .^{\circ} 148$.

Vide $I O M, \mathrm{n} .^{\circ} 12$.

6. Ampurias (Gerona). CIL II, 6183.

Vide $I O M, \mathrm{n} .{ }^{\circ} 25$.

\section{SIGNA}

1. Villalis (León). T. Mañanes, $n .{ }^{\circ} 117$.

Vide $I O M, n{ }^{\circ} 4$.

2. Castro de San Cristóbal, Villalís (León). EPRLe. 38. Vide $I O M, \mathrm{n} .{ }^{\circ} 6$.

3. Villalís (León). CIL II, $2556=\mathrm{T}$. Mañanes, $n .^{\circ} 115$.

Vide $I O M, \mathrm{n} .^{\circ} 7$.

4. Villalís (León). CIL II, $2553=\mathrm{T}$. Mañanes, n. 116.

Vide $1 O M, \mathrm{n}^{\circ} 8$.

5. Villalís (León). T. Mañanes, $n .^{\circ} 119$.

Vide $I O M, \mathrm{n} .{ }^{\circ} 11$.

\section{TITVLI PRO SALVTE IMPERATORIS SACRVM}

1. Luyego de Somoza (León). ILS, $5945=\mathrm{T}$. Mañanes, $n \cdot{ }^{\circ} 112$. Vide $I O M, n .^{\circ} 3$.

2. Villalís (León). T. Mañanes, $n .^{\circ} 117$.

Vide $I O M, \mathrm{n} .{ }^{\circ} 4$. 
3. Villalís (León). T. Mañanes, $n .^{\circ} 114$.

Vide. $I O M, \mathrm{n} .{ }^{\circ} 5$.

4. Castro de San Cristóbal, Villalís (León). EPRLe, 38.

Vide $I O M, \mathrm{n} .{ }^{\circ} 6$.

5. Villalís (León). CIL II, $2556=\mathrm{T}$. Mañanes, $\mathrm{n} .^{\circ} 115$.

Vide $I O M, \mathrm{n} .^{\circ} 7$.

6. Villalís (León). $C / L$ II, $2553=\mathrm{T}$. Mañanes, $\mathrm{n}^{\circ} 116$.

Vide $I O M, \mathrm{n} .{ }^{\circ} 8$.

7. Luyego de Somoza (León). T. Mañanes, n. 113.

Vide $1 O M, \mathrm{n}^{\circ} 9$.

8. Villalís (León). T. Mañanes, $n .^{\circ} 118$.

Vide $I O M, \mathrm{n} .^{\circ} 10$.

9. Villalis (León). T. Mañanes, $n \cdot{ }^{\circ} 119$.

Vide $I O M, \mathrm{n} .^{\circ} 11$.

10. Castro de Corporales (León). T. Mañanes, n. ${ }^{\circ} 148$.

Vide $I O M, \mathrm{n} .^{\circ} 12$.

11. Villalís (León). AE. 1965, $66=T$. Mañanes, $n{ }^{\circ} 120$.

Vide $I O M, \mathrm{n} .^{\circ} 13$.

12. Luyego de Somoza (León). T. Mañanes, n. ${ }^{\circ} 145$.

Vide $I O M, \mathrm{n}^{\circ} 14$.

13. Ruiforco de Torío (Garrafe de Torío, León). CIL II, 2663 = ILS $2335=$ P. Le Roux: L'Armée, n. ${ }^{\circ}$ 253. 25-septiembre-216 ${ }^{67}$.

In centro: IMP(eratori) - CAESARI/ M(arci) - AVREL(i) ANTO/NINO PIO FELI/CI - AVG(usti) - PARTHIC(o)/MAX(imo) - BRIT(anico) - MAX(imo)/ GERMAN (ico) - MAX(imo)/ PONTIF(ice) - MAX(imo)/ TRIB(unicia) .

67 P. Le Roux: L'Armée..., p. 280 piensa en el dies imperii de Caracalla. 
POT(estate) - XVIIII/ CO(n)S(uli) - IIIIIMP(eratori) - III/ P(atri) · P(atriae) . PROC(onsuli)/ EQVITES . IN/ HIS - ACTARIVS/ LEG(ionis) . VII . GEM(inae)/ ANT(oninianae) · P(IAE) · FEL(icis) · DE/VOTI(ssimi) · NVMINI/ MAIESTAQ(Ue) - EIVS.

In latera: DEDICATIVM]/VII K(alendas) OCT(obris)/CATTIO SAB/INO II ET CO[RN(elio)]/ ANVLLINO CO(n)S(ulibus).

14. León. AE. 1911, 94.

Vide IVNO et MINERVA, n. ${ }^{\circ}$ 1. 10-junio-227.

15. Ermita de San Esteban (León). CIL II, 2664. 234.

[IVLIAE MAMMEAE AVG(ustae) MATRI/ AVG(usti)] N(ostri) ET CASTRORVM/ [ET SENATVS] AC PATRIAE/ [- . . - -] F(ilius) . SABATINA/ [P(rimus) · P(ilius)] LEG(ionis) · VII · G(eminae) - SEVER(ianse)/ [ALEXANDR(ianae) .] P(iee) - F(elicis) - EX CORNI/[CVLARIO PRAEF(ecti) PRIAET(Orii) EEM VV/ [MAXIMO II] ET VRBANO CO(nS(ulibus).

16. Rosinos de Vidriales (Zamora). $A E, 1976,288=P$. Le Roux. L'Armée, n. ${ }^{\circ} 255=\mathrm{T}$. Mañanes, $\mathrm{n}{ }^{\circ}$ 133. Int. 251-253.

- - - -/ II . PROICO(n)S(uli) A(la) - II · F(lavia)]/ GALLIANA VOLVS[\&IA]/NA DEVOTA NVMI/[NI] MAIESTAQVE/[EO]RVM.

17. San Pedro de la Viña (Zamora). $A E, 1967,237=T$. Mañanes, $n .^{\circ}$ $132=$ P. Le Roux: L'Armée, . $^{\circ} 252=$ G. Alföldy: Fasti Hispanienses. Senatorische Reichbeamte und Offiziere in der. Spanischen Provinz des Römisches Reiches von Augustus bis Diokletian. Wiesbaden 1969. pp. $90-$ 92. 197.

- - - / [ - - -]l O I[- - -]/O SEVERI AVG(usti)/TRIB(unicia) P(otestate) $V \cdot C O$ (nsulis) II PR[O]/CO(n)S(uli) - STATIVA]M NOVAM/EI AL (a) . II . FL (avia) - H(ispanorum) · C (ivium) - / R(omanorum) - NVMINII]/ EIVS DEVOTISSIMA A[E]/RE CONLATO STATV/EMDAM DECREVERE/RAT . INVICTI · AVG(USti)/ SVA PECVNIA E[RIGI]/IVSSERV[NT].

¿Dies Imperii Septimio Severo? (P. Le Roux, p. 280).

18. Sta. Comba de Bande (Orense). CIL II, $2529=H E p, 2,1990,513$. Int. 210-212.

- - - - [P]RO SALVT[E/ I]VLIAE AV/[G(Ustae)] MATRI/ CASTROR (um)/ ET AVGG(ustorum) LV[CI]/DIVS MA/\{S\}RINVS/ [P]R(aefectus) [Oo] (cohortis) DE/DICAVIT. 


\section{TITVLI MARTI SACRVM}

1. Locus incertus in Gallaecia. $C / L ~ I I, 2600=A E .1963,17.1 .^{\text {a }} 1 / 2 \mathrm{~S}$. II.

MA[RT]I - PATRII TIB(erius) · IVNIVS/TIB(erii) - F(ilius) · QVIR(ina)/ QVADRATVS/ DOMO ROMA/ PRAEF(ectus) - EQVIT(um) - ALAE/ II

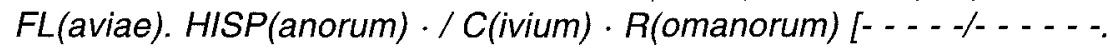

2. Espiño, concejo de Oimbra (Orense). HEp., 2, 1990, 567. S. 1.

MARTI/ S(ulpicius vel sacrum?) VELINV/[S] ALAE/ [- - ] DE S(ua)/ [P(ecunia) P(osuit)] V(otum) [S(olvit) L(ibens) M(erito)].

3. Añavieja (Soria). ILER, $251=$ P. Le Roux: L'Armée, n. ${ }^{\circ} 251$. S. I.

MARTI G(aius) PETRON/VS MATERN/VS Q(uinti) F(ilius) MISSICIVS/ $V$ (otum) S(olvit) L(ibens) M(erito).

4. Nivatia (Baena, Jaén). $C / L I I, 3337=\mathrm{P}$. Le Roux: L'Armée, $\mathrm{n} \cdot{ }^{\circ} 118$. Int. 212-218.

MARTI SANCTO/SVLPICIVS CILO / MILES LEG(ionis) VII GEM(inae)/ ANTONIANAE/ PIAE FELICIS/ ARAM EX VOTO/LIBENS ANIMO/ POSVIT.

\section{TITVLI DIANAE SACRVM}

1. León. CIL II, 2660. Int. 162-166.

Frontal. DIANAE/ SACRVM/ Q(uintus) TVLLIVS/ MAXIMVS/ LEG(atus) • AVGVSTI/ LEG(ionis) · VII · GEMINAE/ FELICIS.....

Tabula Marmarea. Reverso: AEQVORA CONCLVSIT CAMPI/ DIVISQVE DICAVIT/ET TEMPLVM STATVIT TIBI/ DELIA VIRGO TRIFORMIS/ TVLLIVS. ELIBYA. RECTOR/ LEGIONIS HIBERAE (sic)/ VT QVIRET VOLVCRIT CAPREAS/VT FIGERE CERVOS/ SAETIGEROS VT APROS VT/EQVORVM SILIVICOLENTIVM/ PROGENIEM VT CVRSU CERTARE/VT DISICE FERRI/ET FEDES ARMA GENERISET/ EQVO IA CVLATOR HIBERO.

Der.: DENTES APRORVM/ QVOS CECIDIT/MAXIMVS/ DICAT DIANE/ PVLCHRVM VIR/ TUTIS DECVS.

IZda.: CERVOM ALTIFROM/ TVM CORNVA/ DICAT DIANNAE/ TVLLIVS/ QVOS VICIT IN PA/ RAMI AEQVONE/VECTVS FEROCI/ SONIPERE DONAT HAC PELLI DIIANA]/ TVLLIVS TE MA[XIMVS]/ RECTOR AENEANDVM [GEMELLA]/ LEGIO QVIES SE[PTIMA]/IPSE QVAM DETRAIXIT - . - - ] LAVDE OPI[- . - . . . 
2. San Román de Cervantes (Lugo). ILER, $1014=P$. Le Roux: L'Armée, $n^{\circ}$ 37. Int. 41-63.

[DIAN]AE/ SACRVM/C(aius) · VALERIVS/CARVS/MIL(es) LEG(ionis) $X G($ eminae)/V(otum) S(olvit) L(ibens) M(erito).

3. Petavonium (Rosinos de Vidriales, Zamora). R. Martín Valls, M. V. Romero Carnicero y S. Carretero Vaquero: «Aras Votivas de Petavonium. Zephyrus XLVIII, 1995, pp. 337-340, n. ${ }^{\circ}$ 2, 1․ 1/2. S. II.

[SA]NCTAE/ [DIAN]AE . TI(berius) IVN(ius)/ [TI(berii) F(ilius)] QVIRINA/ [Q]VADRATVS/ [POM]O ROMA / [PR(aefectus) EQ (uitum) AL(ae) II FL(aviae)?].

4. Petavonium (Rosinos de Vidriales, Zamora). R. Martín Valls, M. V. Romero Carnicero y S. Carretero Vaquero: «Aras Votivas...», pp. 340-342, n. ${ }^{\circ} 3,2 .^{\text {a }} 1 / 2$. S. 11 .

DIANAE AV̂V (USTa)/ [- A]RRIVS/ [CDINSTANS/ SPERATIANVS/ PRAEF(ectus) EQ(itum) SIGN(ifer) VENATOR/ LIO (verius) EX IVJOTO/ POSVIT.

\section{TITVLI FORTVNAE SACRVM}

1. Rosinos de Vidriales (Zamora). $A E, 1937,166$. S. II.

L(ucius) - VERSENVS/APER - PRAEF(ectus)/ ALAE OPERE/BAL/NEI . SVB SE/ INCOHATO ET/ CONSVMMA/TO FORTVNAE/ L(ibens) V(oto) S(olvit).

2. Ciudadela (La Coruña). AE. $1986,387=$ CIRG I: La Coruña, $n .^{\circ} 31$ = P. Le Roux: «Bilan». p. 253, n. ${ }^{\circ} 20$. S. "I-11.

FORTV/N $\angle A>E$ VAL(erius)/ LVPVS/ OPT(io)/ V(otum) . S(olvit) . $L$ (ibens) M(erito).

3. Vxama (Soria). $A E .1914,21=$ P. Le Roux: L'Armée, $\mathrm{n}^{\circ}$ 117. S. II.

[F]ORTIV]NAE/Q(uintus) C[AECI]LIVS/ TER[TI]VS/M(iles) [L(egionis)] VII G(eminae) F(eLicis)/V(otum) S(olvit) L(ibens) M(erito).

4. Duratón (Segovia), CIL, II, $2763=$ P. Le Roux: L'Armée, n 176, 2. a 1/2. S. I.

FORTVNAE/ BALNEARI/ SAC(rum) Q(uintus) VALE/RIVS TVC/CO MILES/ LEG(ionis) I/ ADIVTRICIS P(iae) F(idelis)/ ) (centuriae) AEMILI(i) S/ECVNDI. 


\section{TITVLI NIMPHIS SACRVM}

1. León. CIL, II $5084=5676=$ P. Le Roux: L'Armée, p. 279. c. 140.

NYMPHIS/ FONTIS - AMEVIIJ/ GN(eus) · L(ucius) TERENTIVS/ L(ucii) F(ilius) HOMVLLVS/IVNOR LEG(atus)/LEG(ionis) VII · G(eminae) · F(elicis).

2. León. $A E .1953,266=\mathrm{P}$. Le Roux: L'Armée, $\mathrm{n}^{\circ}$ 212. S. II.

NIMPPHIS (sic)/ SACRVM/ Q(uintus) · CORNELIVS/ ANTEROS IM/AGINIFER LEG(ionis) VI/ G(eminse) F(elicis)/ CVM VEXIL $<L>A T I / O N E$ $V($ otum $) \cdot S$ (olvit) $\cdot L$ (ibens) $\cdot M($ erito $)$.

3. Torrejoncillo del Rey (Cuenca). P. Le Roux: L'Armée, $n^{\circ} 104$ bis. S. 11. G(aius) MALI(us) VRSSV(s) (sic)/ M(iles) LE(gionis) VII G(eminae) F(elicis)/ [C(ohortis ?)] VIII/ NYMPHIS V(otum) S(olvit) L(ibens) M(erito).

4. Prope Caldas de Reis. ILER, 985. S. II.

T(itus) IVN[IVS SE]/ CVNDV[S MIL(es ?) LEG(ionis)]/ VII GEM(inae) [F(elicis) - - - ]/ V(otum) S(olvit).

\section{TITVLI GENIIS SACRVM}

1. Astorga (León) $A E, 1987,611$. S. II.

[GENIO-] ASTVRIC/[ENSI]VM · CAE/[SIA]NIVS · AG/[RI]COLA · EQ(ues)/ [AL(ae) vel COH(ortis)] · FL(aviae) · I LVSIT(anorum)/ [CJVRATOR.

2. León. $C / L I, 5083=I L S, 2289=$ G. Alföldy: Fasti, p. $119=$ P. Le Roux: L'Armée, p. 278. S. II.

GENIO/ LEG(ionis) · VII [G(eminae) F(elicis)]/ L(ucius) ATTIVS/ MACRO LEG(atus) · AVG(usti).

3. León. $A E .1971,208=1974,411$. 1. 1/2 S. II.

GENIO L(egionis)/ VII G(eminae) - F(elicis) - TI[B(erius)]/ CL(audius) POM/PEIANVS T(ribunus laticlavius)/ [E]XIV(ssu) - G(enii) - S(olvit).

4. Astorga (León). $C / L$ II, $2634=\mathrm{T}$. Mañanes, n. ${ }^{\circ} 12$. Vide $1 O M, \mathrm{n} \cdot{ }^{\circ} 27$ ...GENIO PAETOR(ii)/... Septimio Severo-Clodio Albino (193-197).

5. FALSAE. CIL II, p. 807.

[G]ENII]O/CASTRORVM/- . - 
6. Sta. M. ${ }^{\mathrm{a}}$ de Abedes, Verín (Orense). CIL II, 2522. S. III.

[G(enio)] P(opuli) R(omani)/ C(aius) MAR(cius) MAXI/[M]V[S] LEG (atus)/ [LEG(ionis)] VII G(eminae) P(iae) F(elicis)/ V(otum) S(olvit) L(ibens) $M($ erito).

7. Sta. Eufemia de Tui en San Bartolomé de Rebares (Pontevedra). CIL II, 5613. S. II.

- - - [- . - ] CAEPOL [- - - ET GENIO]/ CONV[ENTI BRACARAVG(ustanorum)]/ TI(berius) - CLAVDI[VS CLAVDIANVS - PRAEF (ectus)] $C<O>H O$ (ortis) BRA[C]A<RA $>$ / AVGVST(anorum) STATVAM/ AVREA[M DEDIT DEDICAVITQVE].

8. Segisamo, Gerion, 10, 1992, pp. 259-273 = CIL II, $2915=$ HEpig, 4, 1994, n. ${ }^{\circ} 201$. Fin s. II. Vide IOM, n. 27.

... ET GENIO] S/[T]ATIONIS · SEGI/SAMONENSIVM...

\section{TITVLI NVMINI SACRVM}

1. Tugia. CIL II, $3327=\mathrm{P}$. Le Roux: L'Armée, n. ${ }^{\circ}$ 256. Vide $/ O M$, número 26.

2. Saldanha (Miranda do Douro, Portugal). AE. 1987, 596. S. I.

I (nvicto) B(eterano) (sic) C(aii) ANTO(nii)/G(allicae) V (legionis)/ NVMINI.

\section{TITVLI ALIIS DIIS SACRVM}

1. Logroño. $C / L$ II, $5810=A E .1903,235=P$. Le Roux: L'Armée, $n .^{\circ}$ 186. S. III.

MERCVRI/ CO<M>PETALI/ FLAVIVS/ FLAVINVS/ VETERANVS/ $V$ (otum) S(olvit) L(ibens) M(erito).

2. Rosinos de Vidriales (Zamora). AE. 1963, 16. Fin s. II- prin. S. III.

HERCVLI . SACRVM/ M(arcus) · SELLIVS - L(ucii) - F(ilius) . ARN(iense) HONORATVS - DO/MO . CHOBA - EX - PR/OVINCIA - MAVR [E/T]ANIA . CAES(ariense) PR A [E]/F (e c t us s). EQ (u itu m). $A$ (lae) $\cdot \| \cdot F($ laviae) $\cdot H($ ispanorum) $\cdot C$ (ivium) $\cdot R$ (omanorum) $\cdot /$ 
VOTVM - COMPO/S - TEMPLA - ALCIDI/ DEO - A FVNDAME/NTIS. EXTRVXIT.

P. Le Roux. p. 279 piensa que levantó un templo en el campamento.

3. Egitania (Portugal). EE. VIII, $14=I L S, 4510 a=P$. Le Roux: L'Armée, n. ${ }^{\circ} 77$. S. I.

[T]ONCIVS/ [T]ONCETAMI/ F(ilius) MILES/ SIGNIFER/ COH(ortis) II LVS(itanorum)/ VIQTORIAE (sic)/ V(otum) S(olvit) L(ibens) M(erito)/ ARDVNNVS/ COMINI F(ilius) FE(cit).

Hay dedicada otra ara por este señor a una divinidad indígena tal vez equivalente a Victoria.

4. Lugo. $C / L$ II $2572=A E .1973$ 393. S. II-III.

AVGG(ustis) SA/CRVM LARIBVS/ VIALIB(us) · M(arcii) · ANNII . VERVS/ ET VERIANVS/)) (centuriones) . LEGG(ionis) PA/TER ET FILIVM/ EX VOTO.

5. Astorga (León). CIL II $2634=\mathrm{T}$. Mañanes, n. ${ }^{\circ} 12$.

Vide IOM, n. ${ }^{\circ} 2$... SOLI INVICTO LIBERO PATRE...

6. Petavonium (Rosinos de Vidriales, Zamora). R. Martín Valls, M. V. Romero Carnicero y S. Carretero Vaquero: «Aras Votivas...». pp. 331-334, n. ${ }^{\circ}$ 1. 2. $2^{\mathrm{a}}$. S. 111 .

NEMESINO(tum) $\cdot S O L$ (vit)/REBVRRV/S.

\section{TITVLI DIIS ORIENTALIS SACRVM}

1. León. $A E, 1967,223$. SS. $\|-\|$.

[AE]SCVLAPIO/ SALVTI/ SEFIAPIDI · ISIDI/ L(UCIUS) · CASSIVS PAVLLVS/ AVGVSTANIVS ALFINVS/ BELLICVS SOLLERS/ ET M(arcus) . CASSIVS AGRIPPA/ SANCTVS . PAVLLINVS/ AVGVSTANIVS $\cdot A L P<I>N V S$.

2. Mérida (Badajoz). M. a A. De Francisco Casado: El Culto de Mithra en Hispania. Granada, 1989, p. 52, n. ${ }^{\circ}$ 1. Año 155.

ANN(o) COL(onise) CLXXXI ARAM GENESIS/ INVICTI MITHRAE/ M(arcus) VAL(erius) SECVNDVS/ FR(umentarius) LEG(ionis) VII GEM(inae folicis) DO(no)/ PONENDAM MERITO CVRAVIT/G(aio) ACCIO HEDYCHO PATRE. 


\section{TITVLI DIIS POPVLORVM HISPANIAE SACRVM}

1. El Barco de Valdeorras (Orense). HEp., 3, 1993 n. ${ }^{\circ}$ 272. S. III.

ATILAECO/ L(ucius) . COR/NELIVS/ PLACID/VS .) (centurio) . LEG(ionis)/VII · [GE(minae)] - P(ise) - F(elicis)/ EX . VOTO - M(erito).

2. Sta. María de Condesado Sarcaus (Orense). CIL II 2515. S. II.

- . . . / . . . - SVLPI]CIVS/ [MIL(es ?) LE]G(ionis) · VII · <G(eminae)> $F$ (elicis) - BA/[N]DV $<A>E$ AE/TOBRIGO/ V(otum) - S(olvit) - L(ibens) . $M($ erito)

3. Rairiz de Vega (Orense). $A E .1967230=A E .1968$ 237. S. II. $V$ (otum) - S(olvit) · L(ibens) - M(erito)/ BANDV $\angle A>E / V E I G E B R / E A G O /$ M(arcus) - SILONIIVS · GAL(eria) · SI/LANVS/ SIG(nifer) · COH(ortis) · I . /GALL(orum) $\cdot C$ (ivium) $\cdot R$ (omanorum).

4. Longroiva Meda (Portugal). AE. 1985524 = P. Le Roux: «Bilan».p. 257, n. ${ }^{\circ} 35.1 . \stackrel{a}{1 / 2}$ S. I. Cfr..$^{\circ} 2$ y 3.

Q(uintus) IVLIVS/MONTA[NV]S/ EQ(ues) LEG(ionis) VII G(eminae)/ F(elicis) BANDI [L]O/NGOBRICV(m)/ V(otum) S(olvit) L(ibens) M(erito).

5. Peña escrita en la Feligresía de Vilar de Perdices concejo de Montalque Vila Real (Portugal). HEp., 2, 1990, 874. S. III.

Cara 1: I (ovi) SOC(io) LAROCVO.

Cara 2: LA[ROC]VO [ - - ]/ M(iles) $L E G$ (ionis) VII $<G$ (eminse) $>P$ (iae) $F($ elicis) ) (centuriae) [- - - - ].

Cara 3: MII [- - - -]ORVO.

Cara 4: $O[---]$.

Cara 5: R [- - - ] CROSV [- . - ] L L - - ]/ ATHX [- - - ].

6. Corneces Pquía. de San Martín de Cornetes concejo de Amoeiro (Orense). HEp., 2, 1990, 571 = P. Le Roux: L'Armée, n. ${ }^{\circ} 87=I L E R, 5997$. 29-mayo-79.

Cara 1: MOELIO/ MORDO/NIEGO/ L(ucius) CAECILINSS FVSCIVS

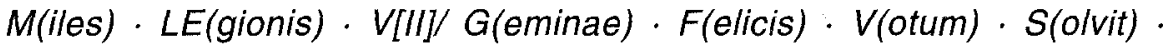
$L$ (ibens) - M(erito).

Cara 2: III K(alendas) IVN(ias)/ II]MP(eratoris) VESP(asiano) VIIII . <ET > TITO VIII]/ CO(n)S(ulibus). 
7. Sto. Tirso. Oporto (Portugal). CIL II $2374=5651=$ P. Le Roux: L'Armée, $\mathrm{n}{ }^{\circ}$ 41. S. I antes del 70.

L(uCius) · VALERIVS · SILVANVS/ MILES · LEG(ionis) · VI · VICT(ricis)/ [DEO ?] TVRIACO/ [V(otum)] $\cdot S($ olvit $) \cdot L$ (ibens) $\cdot M($ erito $)$.

8. Egitania (Portugal). EE. VIII $15=I L S, 4510 . S$. I.

ARA(m) POS(uit)/ TONCIVS/ TONCETAM(i)/ FICAEDIT(onum)/ MILIS (sic)/ TREBARVN $\angle A>E / L$ (ibens) M(erito) V(otum) S(olvit).

9. Poza de la Sal (Burgos). CIL II $746=$ P. Le Roux: L'Armée, n. ${ }^{\circ}$ 185. S. II.

SATTVNIO DEO SAC(rum)/ L(UCiuS) AVFIDIVS MASCVLLINVS/ $S E<X>$ QVIPLICARIVS/ $P$ (raefecti vel primi?) P(rastorii vel pili?) FAC(iendum) CVR(avit).

10. Viseo (Portugal). CIL II $403=$ P. Le Roux: L'Armée, n. ${ }^{\circ}$ 191. S. II.

[- - - -] DEA[E] CABAR[DIACENSIS]/SVL[P (icius) AVITVS/ [M]IL(es) [IMA]GINIFER C<O>HOR(tis) III [G]AL(lorum) CENDT[- - - - -]ESTI $V$ (otum) L(ibens) A(nimo) S(olvit).

11. Mago (Ciudadela Mahón). ClL II $3716=\mathrm{P}$. Le Roux: L'Armée, n. ${ }^{\circ}$ 264. S. I.

LVNAE/C(aius) IVLIVS/ FLAVI<V>S/MILES/V(otum) S(olvit) L(ibens) $M$ (erito). 